\title{
Love wave in a classical linear elastic half-space covered by a surface layer described by the couple stress theory
}

Received: 21 February 2018 / Revised: 18 September 2018 / Published online: 25 October 2018

(C) The Author(s) 2018

\begin{abstract}
A Love wave is derived for a new physical configuration in which a surface layer described by the couple stress theory covers a classical elasticity half-space. The dispersion equation is derived analytically when the thickness of the surface layer approaches zero. The correctness of the dispersion equation is confirmed via the second derivation path, namely the surface elasticity. The membrane with microstructure is described by the surface elasticity which significantly simplifies the derivation. New propagation features deduced from the dispersion curves are discussed.
\end{abstract}

\section{Introduction}

Our interest in the present propagation model is motivated by the applications of the micro-patterns/microstructures on surfaces of micro-electromechanical systems (MEMS). We may model the surface layers with microstructures on top of a homogeneous substrate. In general, the surface layer and the substrate material have their own characteristic microstructure in different length scales. We consider the case in which the surface pattern is a man-made microstructure which may be of the order of micrometers, while the substrate micro-length scale is of the order of nanometers. Therefore, in our physics modeling, the substrate material is treated by the classical linear elasticity where the micro-length is negligibly small.

On the other hand, the surface layer is treated by the couple stress theory (or the higher-order strain gradient theories) where a micro-length is explicitly present in the formulation. The above physical modeling is reasonable and necessary when a surface wave length is of the same order of the surface layer micro-length, but still much larger than the micro-length of the substrate material.

The couple stress theory is a starting point of the general strain gradient theories. Research on the couple stress has a relatively long history. We made our search for the related references from 1960s. The representative contributions to the couple stress theory by Mindlin and Tiersten [1] and Toupin [2] among others. Since then, the research activities have been following two directions. The first direction is to develop more comprehensive strain gradient theories (e.g., Mindlin [3], Mindlin and Eshel [4], Lam et al. [5], and Askes and Aifantis [6] to name a few). In these higher-order strain gradient theories, the couple stress theory takes a special place. The second research direction is to modify the couple stress theory since it is still attractive in its clear physical meaning and relative simplicity (for example, in terms of a number of micro-length parameters) as compared with higher-order theories. The couple stress theory has been re-examined and modified by many researchers

H. Fan $(\bowtie)$

School of Mechanical and Aerospace Engineering, Nanyang Technological University, Singapore 639798, Republic of Singapore

E-mail: mhfan@ntu.edu.sg

L. Xu

School of Aeronautics and Astronautics, University of Electronic Science and Technology of China, Chengdu 611732,

People's Republic of China

E-mail: xulimei@uestc.edu.cn 


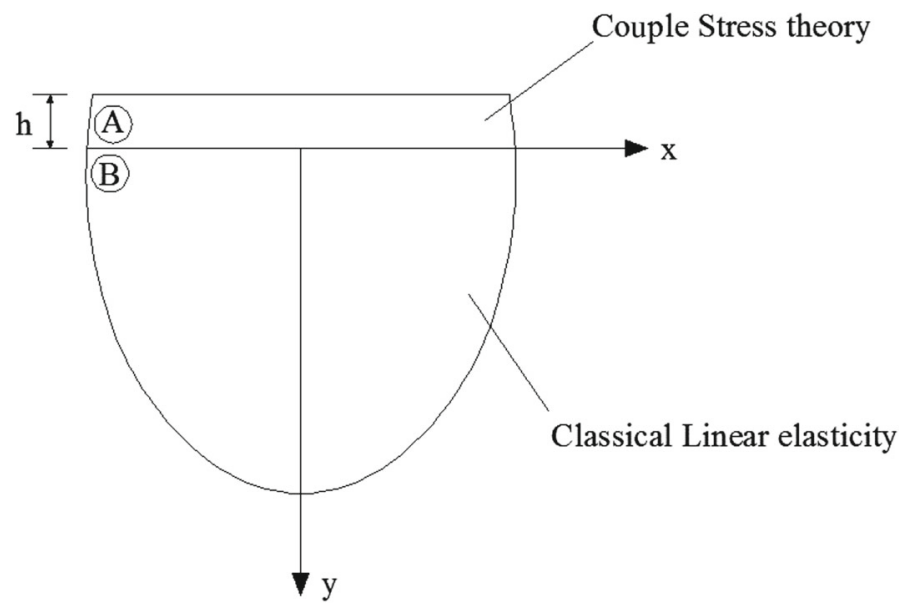

Fig. 1 The anti-plane configuration of a couple stress described surface layer covered on a classical linear elasticity half-space

since 1960s. Recent contributions are, for example, due to Yang et al. [7], Hadjesfandiari and Dargush [8], and Neff et al. [9] to name a few. Certain issues in the couple stress theory have been under debate and not settled. In the following Sections, we will adopt the original couple stress theory derived by Mindlin and Tiersten [1]. A new feature of Love wave is expected from this simplest version of the gradient theories.

In the present study, we consider the horizontal shear $(\mathrm{SH})$ wave in a classical linear elastic half-space covered by a surface layer described by the couple stress theory. In a homogenous classical linear elastic half-space, the decaying surface SH wave does not exist. By adding a surface layer with a different modulus from that of the half-space, the surface SH wave decaying from the free surface is possible and has been called Love wave (e.g., Achenbach [10]). Nevertheless, the decaying SH wave in a homogeneous half-space may be possible under a certain strain gradient theory. Vardoulakis and Georgiadis [11] and Gourgiotis and Georgiadis [12] derived the decaying surface SH wave in a homogeneous half-space without a surface layer via a version of the strain gradient theory. Their solutions showed that the existence of the decaying surface SH wave in the homogeneous half-space under the strain gradient theories depended on the adoption of the micro-inertia in the kinetic energy [3]. Opposed to approaches presented in [11,12], our objective in the present paper is to construct Love waves for the geometric and physical configuration shown in Fig. 1, where the surface layer is treated by the couple stress theory and the half-space is treated by the classical linear elasticity. The solution can be simplified under the condition that the thickness of the surface layer is very thin. More insights in analytical forms are expected after the above thin membrane simplifications. Furthermore, we derived the dispersion equation from the second pathway by using the surface elasticity concept (Gurtin and Murdoch [13]). The comparison between the full version solution and the surface elasticity solution gives the guidance how to apply the surface elasticity incorporated with the couple stress theory to more complex boundary value problems.

\section{The couple stress theory}

Let us follow Mindlin and Tiersten's derivation and notation [1]. The governing equations are briefly presented in this Section. Readers are referred to Mindlin's original paper for the detailed derivation and explanations. The governing equations in tensor notation are classified into the following three groups.

(i) Equilibrium equations, namely the force and couple equilibrium equations:

$$
\begin{aligned}
& \nabla \bullet \tau+\mathbf{f}=\rho \ddot{\mathbf{u}}, \\
& \nabla \bullet \mu+\mathbf{c}+\tau \mathbf{X I}=\mathbf{0}
\end{aligned}
$$

where $\tau$ is the force stress and $\mu$ is the couple stress. It should be pointed out that the force stress tensor is not a symmetric tensor in the couple stress theory. 
(ii) Kinematics equations for small deformation:

$$
\begin{aligned}
& \boldsymbol{\varepsilon}=\frac{\mathbf{1}}{\mathbf{2}}(\nabla \mathbf{u}+\mathbf{u} \nabla), \\
& \mathbf{w}=\frac{1}{2} \nabla \times \mathbf{u}, \\
& \mathbf{\kappa}=\nabla \mathbf{w}
\end{aligned}
$$

where $\mathbf{w}$ is the rotation vector and $\boldsymbol{\kappa}$ is the rotation gradient tensor.

(iii) Constitutive equations for isotropic solids:

$$
\begin{aligned}
& \boldsymbol{\tau}^{S}=\lambda \boldsymbol{\varepsilon}_{\mathbf{S}} \mathbf{I}+2 G \boldsymbol{\varepsilon}, \\
& \boldsymbol{\mu}^{D}=4 \eta \boldsymbol{\kappa}+4 \eta / \boldsymbol{\kappa}_{\mathbf{C}}
\end{aligned}
$$

where $\tau^{S}$ is the symmetric part of the force stress tensor and $\mu^{D}$ is the deviator part of the couple stress tensor. Also, $\boldsymbol{\varepsilon}_{\mathbf{S}}$ is the spherical part of the strain tensor and $\boldsymbol{\kappa}_{\mathbf{C}}$ the transpose of $\boldsymbol{\kappa}$. For convenience in the following Sections, we may use an alternative form of Eq. (2.7),

$$
\mu^{D}=4 G l^{2}\left(\kappa+\bar{\eta} \kappa_{\mathbf{C}}\right),
$$

where $\bar{\eta}=\frac{\eta^{\prime}}{\eta}$. More importantly, Eq. (2.8) introduced a length,

$$
l^{2}=\frac{\eta}{G}
$$

which is interpreted as microstructure length scale. The material constants in Eqs. (2.6)-(2.8) are taken in the following range:

$$
G>0, \quad \lambda>0, \quad \eta>0, \quad-1<\bar{\eta}<1,
$$

for the strain energy to be positive definite.

If we use the displacement formulation, the governing equation derived from the above Eqs.(2.1)-(2.7) is given by Mindlin and Tiersten [1],

$$
G \nabla^{2} \mathbf{u}+(\lambda+G) \nabla \nabla \cdot \mathbf{u}+\eta \nabla^{2} \nabla \times \nabla \times \mathbf{u}+\mathbf{f}+\frac{1}{2} \nabla \times \mathbf{c}=\rho \ddot{\mathbf{u}} .
$$

To complete the boundary value problem, we need the boundary conditions which are derived from the energy balance as follows:

$$
\int_{V}\left[\frac{1}{2} \rho \dot{\mathbf{u}} \cdot \dot{\mathbf{u}}+W\right]_{t_{0}}^{t} \mathrm{~d} V=\int_{t_{0}}^{t} \mathrm{~d} t \int_{V}\left(\mathbf{f}+\frac{1}{2} \nabla \times \mathbf{c}\right) \cdot \dot{\mathbf{u}} \mathrm{d} V+\int_{t_{0}}^{t} \mathrm{~d} t \int_{S}[\mathbf{p} \cdot \dot{\mathbf{u}}+\mathbf{n} \cdot \boldsymbol{\mu} \cdot(\mathbf{I}-\mathbf{n n}) \cdot \dot{\mathbf{w}}] \mathrm{d} S .
$$

In the last integral of Eq. (2.11), the displacement vector and the modified force traction form the energy conjugated pair. It is defined as

$$
\mathbf{p}=\mathbf{n} \cdot \tau^{\mathbf{S}}+\frac{1}{2} \mathbf{n} \times\left(\nabla \cdot \mu^{D}-\nabla \mu_{n n}^{D}\right),
$$

and $\mu_{n n}^{D}=\mathbf{n} \cdot \mu^{D} \cdot \mathbf{n}$ where the subindices do not make summation in the last equation. Also, the couple stress traction and the rotation vector form another energy conjugate pair in the last integral of Eq. (2.11). An important feature stated in Eq. (2.11) is that the normal component of the couple stress traction is not included in the pairs of conjugated boundary conditions. This fact has been derived, discussed, and debated in detail again and again by various researchers (for example, Mindlin and Tiersten [1], Yang et al. [7], and Neff et al. [9] to name a few). Therefore, there are in total five energy conjugated pairs if we write them explicitly in components. 


\section{Anti-plane waves}

3.1 Anti-plane wave in the surface layer described by the couple stress theory

The general equations presented in Sect. 2 can be significantly simplified for the anti-plane problem as shown in Fig. 1 where

$$
u_{1}=0, \quad u_{2}=0, \quad u_{3}=u_{3}\left(x_{1}, x_{2}, t\right) .
$$

Subsequently, the nonzero components in strain and rotation tensors are identified as

$$
\begin{aligned}
\varepsilon_{31} & =\frac{1}{2} u_{3,1}, \quad \varepsilon_{32}=\frac{1}{2} u_{3,2}, \\
\kappa_{11} & =\frac{1}{2} u_{3,21}, \quad \kappa_{12}=-\frac{1}{2} u_{3,11}, \quad \kappa_{21}=\frac{1}{2} u_{3,22}, \quad \kappa_{22}=-\frac{1}{2} u_{3,21} .
\end{aligned}
$$

The corresponding components of the force stress and the couple stress can be calculated via Eqs. (2.6) and (2.7).

Substituting Eqs. (3.1) into (2.10), we have the governing equation

$$
G_{A} \nabla^{2} u_{3}^{A}-\eta \nabla^{4} u_{3}^{A}=\rho_{A} \ddot{u}_{3}^{A}\left(x_{1}, x_{2}, t\right)
$$

where we labeled the field variables and material properties of the surface layer by the subscript or superscript " $A$ " to distinguish later label " $B$ " for the half-space. The Laplacian operator in Eq. (3.3) is a two-dimensional differential operator in $x_{1}-x_{2}$ coordinate. Referring to Fig. 1, we are looking for the solution to Eq. (3.3) in the following form:

$$
u_{3}^{A}\left(x_{1}, x_{2}, t\right)=A e^{\alpha y} \cos (k x-\omega t) \text { for } \quad-h<y<0 .
$$

By substituting Eqs. (3.4) into (3.3), we have

$$
-k^{2}+\alpha^{2}-l^{2}\left(k^{4}-2 k^{2} \alpha^{2}+\alpha^{4}\right)=-\frac{\omega^{2}}{c_{A}^{2}}
$$

where $c_{A}^{2}=\frac{G_{A}}{\rho_{A}}$ is the shear wave speed traveling in the surface layer materials, and $l^{2}=\frac{\eta}{G_{A}}$. Solving the above equation for $\alpha$, we have four roots as follows:

$$
\begin{aligned}
& \alpha_{1,2}^{2}=-\left[\frac{1}{2 l^{2}}\left(\sqrt{1+\frac{4 l^{2} \omega^{2}}{c_{A}^{2}}}-1\right)-k^{2}\right]=-p^{2}, \\
& \alpha_{3,4}^{2}=\frac{1}{2 l^{2}}\left(\sqrt{1+\frac{4 l^{2} \omega^{2}}{c_{A}^{2}}}+1\right)+k^{2}=q^{2} .
\end{aligned}
$$

Subsequently, we write the solution to Eq. (3.3) in a more explicit form:

$$
u_{3}^{A}\left(x_{1}, x_{2}, t\right)=(A \cos p y+B \sin p y+C \cosh q y+D \sinh q y) \cos (k x-\omega t)
$$

where $p$ and $q$ are defined in Eqs. $(3.5 .1,2)$. The four constants $A, B, C$, and $D$ are to be determined by the boundary conditions and the continuity conditions presented in the following Subsection.

A piece of important information in the above solution embedded in Eq. (3.5.1) must be highlighted. The requirement of

$$
p^{2}=\left[\frac{1}{2 l^{2}}\left(\sqrt{1+\frac{4 l^{2} \omega^{2}}{c_{A}^{2}}}-1\right)-k^{2}\right]>0
$$

posts a constraint on the phase velocity, i.e.,

$$
V^{2}=\frac{\omega^{2}}{k^{2}}>c_{A}^{2}\left(1+l^{2} k^{2}\right) .
$$

Equation (3.8) is an important feature in the dispersion curves shown in Sect. 6. 
3.2 Anti-plane surface decay wave in the classical elastic half-space

The governing equation for the anti-plane wave in a classical linear elastic half-space is well known,

$$
G_{B} \nabla^{2} u_{3}^{B}=\rho_{B} \ddot{u}_{3}^{B}\left(x_{1}, x_{2}, t\right), \quad y>0 .
$$

The subscript and superscript " $B$ " denote the materials properties and the displacement in the half-space. The surface wave to Eq. (3.9) is assumed in the form of

$$
u_{3}^{B}\left(x_{1}, x_{2}, t\right)=F e^{-\beta y} \cos (k x-\omega t) \quad y>0
$$

and

$$
\tau_{23}^{B}=G_{B} \frac{\partial u_{3}^{B}}{\partial y}=-\beta G_{B} F e^{-\beta y} \cos (k x-\omega t) .
$$

Equation (3.10.2) is obtained via Hooke's law and will be needed in the continuity condition presented in the following Subsection.

By substituting Eq. (3.10.1) into (3.9), we have

$$
\beta=\sqrt{k^{2}-\frac{\omega^{2}}{c_{B}^{2}}}=k \sqrt{1-\frac{V^{2}}{c_{B}^{2}}} \leq 0 .
$$

The non-decay solution is dropped out.

Equations (3.11) and (3.8) give us the range of the phase velocity within

$$
c_{A}^{2}\left(1+l^{2} k^{2}\right)<V^{2} \leq c_{B}^{2} .
$$

The constant $F$ in Eq. (3.10.1) is to be determined by the continuity condition in the following Subsection.

\subsection{Boundary conditions and continuity conditions}

To determine the five constants $(A, B, C, D$, and $F$ ) appearing in the above wave solutions, we need to examine the energy conjugated pairs in boundary conditions Eq. (2.11). Along the free surface $y=-h$, we have the traction conditions as follows:

$$
\begin{aligned}
& p_{z}^{A}(x,-h, t)=0, \\
& \mu_{y x}^{A}(x,-h, t)=0 .
\end{aligned}
$$

They are the force traction and the couple stress traction, respectively.

Along the interface between the Solid A and Solid B, $y=0$, we have the displacement and the traction continuity conditions as follows:

$$
\begin{aligned}
& u_{3}^{A}(x, 0, t)=u_{3}^{B}(x, 0, t), \\
& p_{3}^{A}(x, 0, t)=\tau_{23}^{B}(x, 0, t), \\
& \mu_{y x}^{A}(x, 0, t)=0 .
\end{aligned}
$$

Continuity conditions (3.14.1-3) may deserve a few more lines of discussion presented in "Appendix A."

The five conditions in Eqs. (3.13.1) and (3.14.1) will determine five constants $A, B, C, D$, and $F$ in the wave solutions of Eqs. (3.6) and (3.10.1).

By noting Eq. (2.12) and Sect. 5 in Mindlin and Tiersten [1], we have

$$
\begin{aligned}
& p_{3}^{A}=G_{A}\left(u_{3,2}^{A}-l^{2}\left[(2+\bar{\eta}) u_{3.11}^{A}+u_{3,22}^{A}\right], 2\right), \\
& \mu_{y x}^{A}=2 G_{A} l^{2}\left(u_{3,22}^{A}-\bar{\eta} u_{3,11}^{A}\right) .
\end{aligned}
$$


Substituting Eqs. (3.6) into $(3.15 .1,2)$, we have the boundary conditions at $y=-h$, Eqs. $(3.13 .1,2)$, explicitly as

$$
\begin{aligned}
& p\left(1+(2+\bar{\eta}) l^{2} k^{2}+l^{2} p^{2}\right) A \sin p h+p\left(1+(2+\bar{\eta}) l^{2} k^{2}+l^{2} p^{2}\right) B \cos p h \\
& \quad-q\left(1+(2+\bar{\eta}) l^{2} k^{2}-l^{2} q^{2}\right) C \sinh q h+q\left(1+(2+\bar{\eta}) l^{2} k^{2}-l^{2} q^{2}\right) D \cosh q h=0, \\
& \left(\bar{\eta} k^{2}-p^{2}\right) A \cos p h-\left(\bar{\eta} k^{2}-p^{2}\right) B \sin p h \\
& \quad+\left(\bar{\eta} k^{2}+q^{2}\right) C \cosh q h-\left(\bar{\eta} k^{2}+q^{2}\right) D \sinh q h=0 .
\end{aligned}
$$

We also have the continuity conditions at $y=0$, Eqs. (3.14.1-3), explicitly as

$$
\begin{aligned}
& A+C=F, \\
& , G_{A}\left(p\left(1+(2+\bar{\eta}) l^{2} k^{2}+l^{2} p^{2}\right) B+q\left(1+(2+\bar{\eta}) l^{2} k^{2}-l^{2} q^{2}\right) D\right)=-\beta G_{B} F, \\
& \\
& \left(\bar{\eta} k^{2}-p^{2}\right) A+\left(\bar{\eta} k^{2}+q^{2}\right) C=0 .
\end{aligned}
$$

Equations (3.16.1-5) are to be solved for constants $A, B, C, D$, and $F$ presented in the wave solution Eqs. (3.6) and (3.10). Parameters $p, q$, and $\beta$ are given by Eqs. (3.5.1, 2). The derivation of the dispersion equation from Eqs. (3.16.1-5) by taking the determinant of a 5 by 5 coefficient matrix to be zero is seemingly messy. However, it may be workable by the following procedure:

(i) Use Eqs. $(3.16 .1,2)$, represent $B$ and $D$ in terms of $A$ and $C$,

(ii) Use Eqs. $(3.16 .3,5)$, represent $A$ and $C$ in terms of $F$,

(iii) Substitute the above results into Eq. (3.16.4) so that both sides of the equation contain $F$ only.

For a nonzero value of $F$, we need the coefficient of $F$ to be zero, which is the dispersion equation for the new Love wave. However, even though we obtain this dispersion equation, we do not expect an analytical solution. The numerical approach is needed to obtain the dispersion curve in the $V \sim k$ space.

In order to gain further analytical insight, we will examine a simplified case by letting $h \rightarrow 0$ in the above solution. Physically, it means that we are considering a membrane by taking the thickness of the surface layer approaching zero.

\section{The couple stress described surface layer as the thickness is approaching zero}

The lowest order solution for the thickness of the surface layer approaching zero is of our interest as we will take it as a benchmark for our later solution via the surface elasticity. We only keep the first term of the Taylor series expansion of the trigonometric functions in Eqs. $(3.16 .1,2)$. The boundary conditions at $y=-h$ become

$$
\begin{aligned}
& p^{2} h\left(1+(2+\bar{\eta}) l^{2} k^{2}+l^{2} p^{2}\right) A+p\left(1+(2+\bar{\eta}) l^{2} k^{2}+l^{2} p^{2}\right) B \\
& \quad-q^{2} h\left(1+(2+\bar{\eta}) l^{2} k^{2}-l^{2} q^{2}\right) C+q\left(1+(2+\bar{\eta}) l^{2} k^{2}-l^{2} q^{2}\right) D=0, \\
& \left(\bar{\eta} k^{2}-p^{2}\right) A-p h\left(\bar{\eta} k^{2}-p^{2}\right) B+\left(\bar{\eta} k^{2}+q^{2}\right) C-q h\left(\bar{\eta} k^{2}+q^{2}\right) D=0 .
\end{aligned}
$$

We use Eqs. $(3.16 .3,5)$ to obtain

$$
\begin{aligned}
A & =\frac{\left(\bar{\eta} k^{2}+q^{2}\right)}{p^{2}+q^{2}} F, \\
C & =-\frac{\left(\bar{\eta} k^{2}-p^{2}\right)}{p^{2}+q^{2}} F .
\end{aligned}
$$

Using Eq. (4.1.1), we replace $B$ and $D$ in Eq. (3.16.4) by $A$ and $C$ which are given by Eqs. (4.2) and (4.3). With $p$ and $q$ explicitly given by $(3.5 .1,2)$, we derive the dispersion equation from Eq. (3.16.4),

$$
\frac{G_{A}}{G_{B}} h k\left(\frac{V^{2}}{c_{A}^{2}}-\left[1+\left(1-\bar{\eta}^{2}\right) l^{2} k^{2}\right]\right)=\sqrt{1-\frac{V^{2}}{c_{B}^{2}}} .
$$


We are amazed that such a simple equation is obtained after a lengthy derivation from a set of messy equations (Eqs. (4.1. 1, 2), (3.16. 3-5). However, we are not surprised as we have another pathway to derive a similar dispersion equation as Eq. (4.4) from the surface elasticity presented in Sect. 5.

If we use the following dimensionless parameters, normalized by a length scale of $H=\frac{\rho_{A}}{\rho_{B}} h$ :

$$
K=k H, \quad L=\frac{l}{H}, \quad s=\frac{V}{c_{B}}, \quad s_{0}=\frac{c_{A}}{c_{B}},
$$

we have Eq. (4.4) in a dimensionless form of

$$
K\left(s^{2}-\left(1+\left(1-\bar{\eta}^{2}\right) L^{2} K^{2}\right) s_{0}^{2}\right)=\sqrt{1-s^{2}} .
$$

This set of the dimensionless parameters used in Eq. (4.5) is consistent with the notation used in [14] for an easier comparison. Murdoch [14] used classical linear elasticity for both half-space and the surface membrane. His dispersion curve can be obtained from Eq. (4.6) by setting $L=0$, i.e.,

$$
K\left(s^{2}-s_{0}^{2}\right)=\sqrt{1-s^{2}} .
$$

The group velocity, as an extended result from the phase velocity, is defined as:

$$
V_{g}=V+k \frac{\mathrm{d} V}{\mathrm{~d} k} .
$$

To be consistent with Eq. (4.6), we may use the dimensionless version

$$
s_{g}=\frac{V_{g}}{c_{B}}=s+K \frac{\mathrm{d} s}{\mathrm{~d} K} .
$$

Taking the derivative with respect to $K$ on both sides of Eq. (4.6), we obtain

$$
s_{g}=s-\frac{\left(1-s^{2}\right)-2\left(1-\bar{\eta}^{2}\right) L^{2} K^{3} s_{0}^{2} \sqrt{1-s^{2}}}{s\left(1+2 K \sqrt{1-s^{2}}\right)} .
$$

The numerical illustration of Eqs. (4.6) and (4.9) will be presented in Sect. 6 after we derive the dispersion equation from the surface elasticity.

\section{Derivation of the Love wave solution via the surface elasticity}

If the thickness of the surface layer is very small, the surface layer can be treated as a membrane by applying the surface elasticity approach [13]. The surface elasticity addresses a class of boundary value problems where a two-dimensional membrane is in perfect contact with a three-dimensional elastic solid. The connection between the two-dimensional membrane and the three-dimensional solid is built up via the displacement continuity and conversion of the surface traction of the three-dimensional solid to the "body force" acting on the membrane.

In this Section, we take the second formulation pathway to derive the dispersion equation governing Love waves for the above configuration via the surface elasticity proposed by Gurtin and Murdoch [13]. Since it is a lengthy task to develop a general surface elasticity with the couple stress concept, such a development is beyond the scope and length of the present paper. Hereby, we use the concept of the surface elasticity and consider the anti-plane deformation in the surface layer shown in Fig. 1 as

$$
u_{1}=0, \quad u_{2}=0, \quad u_{3}=u_{3}^{M}(x, t) .
$$

The displacement in the surface layer is assumed to be uniform in the thickness direction. Physically, it is a proper simplification for a very thin membrane. The so-called surface elasticity in the classical linear elasticity has been proved to be a successful theory for the boundary value problems where a two-dimensional membrane is in perfect contact with a three-dimensional solid [13]. The present derivation via the surface elasticity examines the Love wave problem from a different angle, which verifies our derivation, especially the boundary and continuity conditions at $y=0$ in the full version solution of the couple stress theory. 
Recall our formulation for the classical linear elastic half-space presented in Sect. 3.2. The boundary condition at $y=0$ involves the membrane. Substituting the displacement given in Eqs. (5.1) into (2.10), we obtain the equation of motion for the membrane,

$$
G_{M}\left(\frac{\partial^{2} u_{3}^{M}}{\partial x^{2}}-l^{2} \frac{\partial^{4} u_{3}^{M}}{\partial x^{4}}\right)+f_{z}=\rho_{M} \frac{\partial^{2} u_{3}^{M}}{\partial t^{2}}
$$

In Eq. (5.2), " $M$ " is used to label the field variables and material properties of the membrane. Particularly, we kept a nonzero body force term as the membrane and the half-space is connected by the body force instead of the traction continuity conditions as we used in Sect. 3. The upper and lower surfaces of the membrane are no longer considered as boundaries anymore. Instead, the tractions acting on the upper and lower surfaces of the membrane are converted to the body forces. In converting Eqs. (2.10) to (5.2), we dropped the "body couple" induced body force term in Eq. (2.10) as the classical linear elastic half-space does not provide this type of surface traction.

The continuity conditions between the membrane under the couple stress theory and the classical linear elastic half-space are built up by

$$
u_{3}^{B}(x, 0, t)=u_{3}^{M}(x, t)
$$

and

$$
f_{z}=\frac{1}{h} \tau_{23}^{B}=\left.\frac{G_{B}}{h} \frac{\partial u_{3}^{B}}{\partial y}\right|_{y=0}=-\frac{\beta G_{B}}{h} \cos (k x-\omega t)
$$

where Eq. (3.10.2) is used.

The membrane thickness " $h$ " is still needed for the dimensional consistence among the terms in the equation of motion of the membrane. It will be absorbed into the dimensionless parameters later on.

Substituting Eqs. $(5.3 .1,2)$, we obtain

$$
\beta=\frac{G_{M}}{G_{B}} h k^{2}\left(\frac{V^{2}}{c_{M}^{2}}-\left(1+l^{2} k^{2}\right)\right)>0 .
$$

Together with Eq. (3.11), the phase velocity is found within the range of

$$
c_{M}^{2}\left(1+l^{2} k^{2}\right)<V^{2} \leq c_{B}^{2}
$$

which is the same as Eq. (3.12) derived via the full version couple stress theory for the finite thickness of the surface layer, if we take " $M$ " as " $A$ " in the equation. Combining Eqs. (3.11) and (5.4), we obtain the dispersion equation as

$$
K\left(s^{2}-\left(1+L^{2} K^{2}\right) s_{o}^{2}\right)=\sqrt{1-s^{2}}
$$

where we used the same set of dimensionless parameters as defined in Eq. (4.5).

Making a comparison between Eqs. (5.6) and (4.6), we found that they are almost identical except that the microstructure parameter in Eq. (5.6) is $L$, while in Eq. (4.6) the microstructure parameter is $L_{\eta}=$ $L \sqrt{1-\bar{\eta}^{2}}$. Nevertheless, Eqs. (5.6) and (4.6) are not completely equivalent because they must be calculated with consideration of Eq. (5.5) [Eq. (3.12)]. We will provide a detailed discussion in Sect. 6 where we show numerical examples. 


\section{Numerical examples and discussion}

(a) $\bar{\eta}=0$

Under this condition, the full version solution Eq. (4.6) and the surface elasticity solution Eq. (5.6) are exactly identical. The dispersion curves for the phase velocity are shown in Fig. 2 for various values of the microstructure length $L$. The corresponding group velocity is shown in Fig. 3. The important observations are listed as follows.

(i) A Love wave is only available within a limited range of wave numbers for the nonzero micro-length $L$.

(ii) The wave exhibits normal dispersion for low wave numbers, and the wave exhibits anomalous dispersion for high wave numbers.

(iii) All dispersion curves are bounded within the region defined by Eq. (5.5).

(b) $\bar{\eta} \neq 0$

At the first glance at the dispersion equations (4.6) and (5.6),

$$
K\left(s^{2}-\left(1+\left(1-\bar{\eta}^{2}\right) L^{2} K^{2}\right) s_{0}^{2}\right)=\sqrt{1-s^{2}},
$$

obtained via the full version of couple stress theory, and

$$
K\left(s^{2}-\left(1+L^{2} K^{2}\right) s_{o}^{2}\right)=\sqrt{1-s^{2}}
$$

obtained via the surface elasticity with the couple stress theory, we may think that they can be solved via the same procedure as long as we group the parameters

$$
L_{\eta}^{2}=\left(1-\bar{\eta}^{2}\right) L^{2}
$$

together as a single parameter in Eq. (6.1). However, this perception is wrong because the above equations are to be solved with an additional condition, i.e.,

$$
s_{0}^{2}\left(1+L^{2} K^{2}\right)<s^{2} \leq 1
$$

which is the dimensionless version of Eqs. (3.12) and (5.5). Let us rewrite Eq. (6.1) as

$$
K\left(s^{2}-\left(1+L^{2} K^{2}\right) s_{0}^{2}\right)+\bar{\eta}^{2} L^{2} K^{3} s_{0}^{2}=\sqrt{1-s^{2}} .
$$

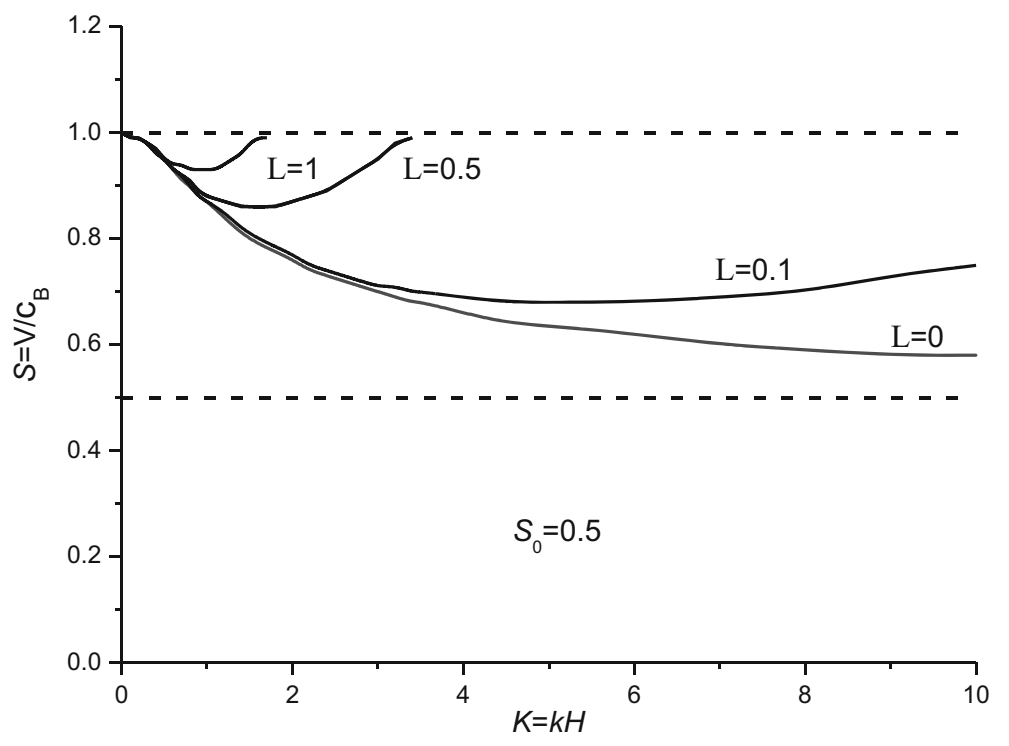

Fig. 2 Normalized velocity dispersion curves for various values of the microstructure parameter with $\bar{\eta}=0$ 


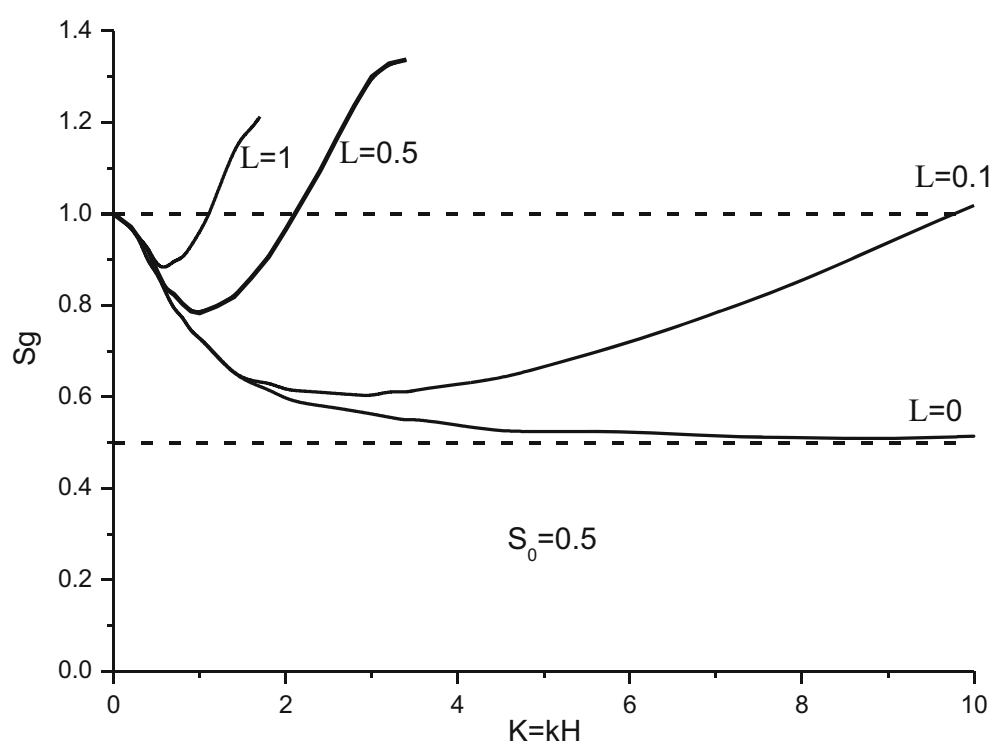

Fig. 3 Group velocity for various values of the microstructure parameter with $\bar{\eta}=0$

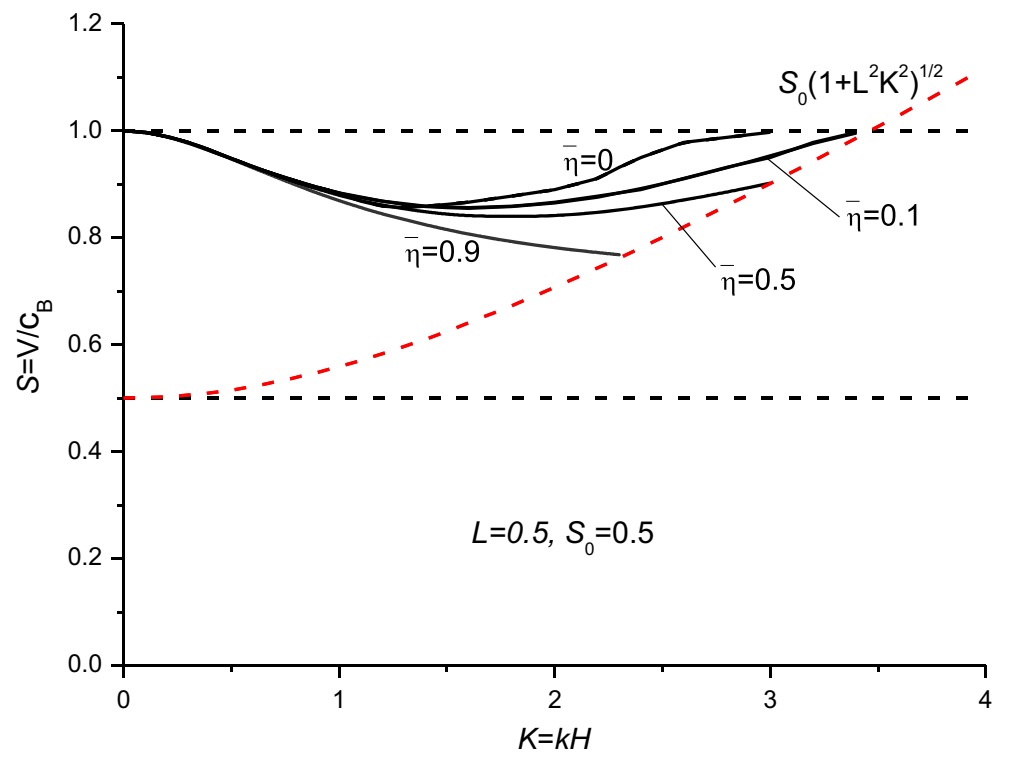

Fig. 4 Effects of parameter $-1<\bar{\eta}<1$ in the couple stress theory

In our numerical calculations, we need to keep all the three terms in Eq. (6.4) to be positive in order to hold condition (6.3) valid. The numerical example for a set of selected parameters is shown in Fig. 4, where Love waves are only available within the region defined by Eq. (6.3).

With these numerical examples in mind, we conclude that the surface elasticity plus the couple stress theory must be used with care. Only when $\bar{\eta}=0$, the two derivations provide an identical dispersion equation. Nevertheless, the solution via the surface elasticity captures the key feature of the boundary value problem as shown in Figs. 2 and 3. Having the solution via the classical linear elasticity, we note that the full version solution after applying the series expansion [10] is identical with the surface elasticity solution [14]. For readers' convenience, we have listed these two classical solutions in "Appendix B" for a quick reference. 


\section{Conclusions}

The present study used two different pathways to derive the dispersion equation of Love waves. The full version solution is rigorous, yet complex and tedious. The surface elasticity solution is simple and has a great potential to be employed in the much more complex multifactor problems (e.g., bi-material interfacial waves and piezoelectric half-space waves). It is one of our original objectives of the present paper that we want to validate the surface elasticity for the future applications in various types of more complex "threedimensional plus two-dimensional" boundary value problems [15-17]. The comparison between these two solutions indicates that these two derivation paths did not give us the same dispersion equations. This situation was not observed in the classical linear elasticity solutions [14]. Nevertheless, the numerical results presented in Figs. 2 and 3 show that the microstructure parameter $L$ gives a new feature of Love waves, i.e., Love waves with $L$ are only available for a limited range of wavelength.

Funding Funding was provided by Key Research and Development Project of Sichuan Provincial Science and Technology Plan, China (Grant No. 2017GZ0162).

\section{Appendix A. Continuity conditions along the interface of solids}

If both of Solid A and Solid B shown in Fig. 1 are the couple stress described solids, the continuity conditions along the interface $y=0$ for the anti-plane deformation, according to Eqs. (2.11) and (2.12), are

$$
\begin{aligned}
& u_{3}^{A}(x, 0, t)=u_{3}^{B}(x, 0, t), \\
& p_{3}^{A}(x, 0, t)=p_{3}^{B}(x, 0, t), \\
& u_{3,2}^{A}(x, 0, t)=u_{3,2}^{B}(x, 0, t), \\
& \mu_{y x}^{A}(x, 0, t)=\mu_{y x}^{B}(x, 0, t) .
\end{aligned}
$$

Among them, Eqs. (A.1.1, 2) form an energy conjugated pair, and Eqs. (A.1.3, 4) form another pair. More explicitly,

$$
\begin{aligned}
& p_{3}^{A}=G_{A}\left(u_{3,2}^{A}-l_{A}^{2}\left[\left(2+\bar{\eta}_{A}\right) u_{3.11}^{A}+u_{3,22}^{A}\right]_{, 2}\right), \\
& \mu_{y x}^{A}=2 G_{A} l_{A}^{2}\left(u_{3,22}^{A}-\bar{\eta}_{A} u_{3,11}^{A}\right), \\
& p_{3}^{B}=G_{B}\left(u_{3,2}^{B}-l_{B}^{2}\left[\left(2+\bar{\eta}_{B}\right) u_{3.11}^{B}+u_{3,22}^{B}\right]_{, 2}\right), \\
& \mu_{y x}^{B}=2 G_{B} l_{B}^{2}\left(u_{3,22}^{B}-\bar{\eta}_{B} u_{3,11}^{B}\right) .
\end{aligned}
$$

As Solid B is approaching a classical linear elastic solid, $l_{B} \rightarrow 0$, the corresponding terms in Eqs. (A.3.1, 2) disappear. The most important outcome of this degeneration is

$$
\mu_{y x}^{A}=2 G_{A} l_{A}^{2}\left(u_{3,22}^{A}-\bar{\eta}_{A} u_{3,11}^{A}\right)=0,
$$

and its energy conjugated partner Eq. (A.1.3) is dropped out from the continuity conditions.

\section{Appendix B. Love wave in classical linear elasticity}

The SH wave solution in the classical elasticity surface layer can be obtained from the original equation of motion, or deducted from the couple stress theory solution (Sect. 3),

$$
u_{3}^{A}\left(x_{1}, x_{2}, t\right)=(A \cos p y+B \sin p y+C \cosh q y+D \sinh q y) \cos (k x-\omega t)
$$

with

$$
\begin{aligned}
& p^{2}=\frac{1}{2 l_{A}^{2}}\left(\sqrt{1+\frac{4 l_{A}^{2} \omega^{2}}{c_{A}^{2}}}-1\right)-k^{2}, \\
& q^{2}=\frac{1}{2 l_{A}^{2}}\left(\sqrt{1+\frac{4 l_{A}^{2} \omega^{2}}{c_{A}^{2}}}+1\right)+k^{2} .
\end{aligned}
$$


To deducte the classical elastic solution from the couple stress theory solution, we take the limit of $l \rightarrow 0$,

$$
\begin{aligned}
& p^{2}=k^{2}\left(\frac{V^{2}}{c_{A}^{2}}-1\right)+O\left(l^{2}\right), \\
& q^{2}=\frac{1}{2 l^{2}}+O(1) \rightarrow \infty .
\end{aligned}
$$

Equation (B.3.2) leads to $C=0$ and $D=0$ in the solution of Eq. (B1), which is the classical elastic solution [10]. The corresponding boundary conditions and continuity conditions associated with couple stress $(3.16 .2,5)$ are dismissed as $l=0$. There are only three boundary and continuity conditions left for determining $A, B$, and $F$.

Examining the difference between the full solution and the surface elasticity solution in the scope of classical linear elasticity, we are able to ascertain the reliability of using the surface elasticity in the couple stress theory. Referring to [10], the dispersion equation is given by the full version solution as

$$
\tan \left[\sqrt{\left(\frac{V}{c_{A}}\right)^{2}-1}\right] k h-\frac{G_{B}}{G_{A}} \frac{\sqrt{1-\left(V / c_{B}\right)^{2}}}{\sqrt{\left(V / c_{A}\right)^{2}-1}}=0
$$

where "A" refers to the surface layer and "B" refers to the half-space. Keeping the first term of the series expansion, we have

$$
\frac{G_{A}}{G_{B}}\left[\left(\frac{V}{c_{A}}\right)^{2}-1\right] k h-\sqrt{1-\left(V / c_{B}\right)^{2}}=0 .
$$

If we use the dimensionless parameter defined in Eq. (4.5), we obtain the exact Eq. (4.7) which was given by Murdoch [14] via the surface elasticity. There is no extra material parameter in the full version solution.

Open Access This article is distributed under the terms of the Creative Commons Attribution 4.0 International License (http:// creativecommons.org/licenses/by/4.0/), which permits unrestricted use, distribution, and reproduction in any medium, provided you give appropriate credit to the original author(s) and the source, provide a link to the Creative Commons license, and indicate if changes were made.

\section{References}

1. Mindlin, R.D., Tiersten, H.F.: Effects of couple-stress in linear elasticity. Arch. Ration. Mech. Anal. 11, 415-488 (1962)

2. Toupin, R.A.: Elastic materials with couple stresses. Arch. Ration. Mech. Anal. 11, 385-414 (1962)

3. Mindlin, R.D.: Micro-structure in linear elasticity. Arch. Ration. Mech. Anal. 16, 51-78 (1964)

4. Mindlin, R.D., Eshel, N.N.: On first strain gradient theories in linear elasticity. Int. J. Solids Struct. 4, 109-124 (1968)

5. Lam, D.C.C., Yang, F., Chong, A.C.M., Wang, J., Tong, P.: Experiments and theory in strain gradient elasticity. J. Mech. Phys. Solids 51, 1477-508 (2003)

6. Askes, H., Aifantis, E.C.: Gradient elasticity in statics and dynamics: an overview of formulations, length scale identification procedures, finite element implementations and new results. Int. J. Solids Struct. 48, 1962-1990 (2011)

7. Yang, F., Chong, A.C.M., Lam, D.C.C., Tong, P.: Couple stress based strain gradient theory for elasticity. Int. J. Solids Struct. 39, 2731-2743 (2002)

8. Hadjesfandiari, A.R., Dargush, G.F.: Couple stress theory for solids. Int. J. Solids Struct. 48, 2496-2510 (2011)

9. Neff, P., Münch, I., Ghiba, I.D., Madeoe, A.: On some fundamental misunderstandings in the indeterminate couple stress model. A comment on recent papers of A.R. Hadjesfandiari and G.F. Dargush. Int. J. Solids Struct. 81, 233-243 (2016)

10. Achenbach, A.D.: Wave Propagation in Elastic Solids. North-Holland, Amsterdam (1973)

11. Vardoulakis, I., Georgiadis, H.G.: SH surface waves in a homogeneous gradient-elastic half-space with surface energy. J. Elast. 47, 147-165 (1997)

12. Gourgiotis, P.A., Georgiadis, H.G.: Torsional and SH surface waves in an isotropic and homogenous elastic half-space characterized by the Toupin-Mindlin gradient theory. Int. J. Solids Struct. 62, 217-228 (2015)

13. Gurtin, M.E., Murdoch, A.I.: A continuum theory of elastic material surfaces. Arch. Ration. Mech. Anal. 57, 291-323 (1975)

14. Murdoch, A.I.: The propagation of source waves in bodies with material boundaries. J. Mech. Phys. Solids 24, 137-146 (1976)

15. Fan, H., Xu, L.M.: Decay rates in nano tubes with consideration of surface elasticity. Mech. Res. Commun. 73, 113-116 (2016)

16. Wang, X., Fan, H.: A piezoelectric screw dislocation in a bimaterial with surface piezoelectricity. Acta Mech. 226, 3317-3331 (2015)

17. Xu, L.M., Wang, X., Fan, H.: Anti-plane waves near an interface between two piezoelectric half-spaces. Mech. Res. Commun. 67, 8-12(2015)

Publisher's Note Springer Nature remains neutral with regard to jurisdictional claims in published maps and institutional affiliations. 